\title{
An Adaptable University Human Resource Data Management
}

\author{
Ye Fan, Shaoyun Guan and Honglue LV \\ Harbin University of Commerce, Harbin, china \\ gsyj91@163.com
}

\begin{abstract}
Data management is the foundation of the University Human Resource Management Systems (UHRMSs). The data collected by the different departments in university human resource systems is distortion, logical confusion and unstructured. In order to overcome these defects, we design an adaptable university human resource management system. In the system, the technology of adaptive computing is adopted to enhance the selfmanagement capability of UHRMS and decrease the complexity of management. Experimental results show that the proposed adaptable UHRMS has a lower transaction response time and a higher throughput than the traditional methods.
\end{abstract}

Keywords: human resource data management; information management system; adaptable; self-management

\section{Introduction}

Recently with the personnel system reform advancing gradually in colleges and universities, management patterns of human resources is constantly changing such as more and more persons, more complex businesses, more dispersed campuses, more frequent movement of personnel and so on. Therefore, human resources management must be more standardized, scientific and automation to improve the management level. Meanwhile the original personnel information scattered in various departments should be organized and managed organically to achieve information sharing [1]. More and more universities make use of the University Human Resource Information Systems (UHRIS) based on B/S (Browser/Server) mode to resolve these problems [2]. It applied web technology and achieved the human resources information central management in dispersed areas. It can operate system anywhere without having to install any special software, so it may reduce significantly the cost of the system's operation and maintenance and improve work efficiency and quality of service. It can also provide data supporting for schools scientific decision-making and finally bring about scientific management of human resources in universities.

However, the more and more complexity of system architecture brings a series of challenges in management, which makes the UHRIS hard to maintain. In the paper, we want to build a novel UHRIS with adaptive capabilities to reduce the management works for administrators.

\section{The Shortcomings of Existing UHRIS}

Data management based on $\mathrm{B} / \mathrm{S}$ mode in university human resource information system is very important because information is openness and person relationship is complexity. It is known that in the field of information system management, perfect technology decides $30 \%$ of the result while excellent management $70 \%$, data $120 \%$. It would undermine seriously the efficiency and the reliability of relevant decisions without correct quality data during university human resource management. It would happen undesirable phenomena that garbage in, garbage out without good UHRIS. 
The existing problems of UHRIS can be summarized as followings.

\section{1) Data missing}

This is one of the most common data problems in human resources information system. The one reason is that the data collected was paid different attentions in different department, and sometimes it is caused without unified norms and standards when the data was input.

\section{2) Data distortion}

Data may be inaccurate and not timely updated. Inaccurate data could be caused by inputting errors or non-standard data but they are not detected. It was also probable that the data which come from different sources was integrated simply without reference to harmonized standards. In other time, such as paper [3], the actual data information changes but it is not updated timely by administrators at all levels, which cause data not to be updated timely either.

3) Data logical confusion

It was caused that data come from different sources. Different business department inputs independent data to system or data connection is not accurate, contradictory when they are rolled up or analyzed. With the continuous expansion of university staff, more than the phenomenon of the same name is also often. It is easy to produce data linkage errors if these same names cannot be distinguished correctly.

4) Unstructured data

Some data do not have clear format in system, each record in inputting data is possible in a different order, such as the addresses can be input according to postal code, province, streets or according to streets, province, postal code. They are decomposed easily to individual property which could not be queried or analyzed by SQL language. Furthermore, unstructured data can be caused while a data item is used for various purposes, that is, those data without relationship are linked together [4], for example, two different business systems write data to the same data file, where intermixed data could not be utilized and judged by users.

5) Threating to security

More and more security threats come from networks because of $\mathrm{B} / \mathrm{S}$ mode. The data protected may be leak or disclose to unauthorized entities, therefore it may suffer unauthorized additions, deletions, modification or destruction. Legal access to data or other resources are blocked unconditionally, while either illegal users disguise as a legal user or users with low privilege get high privilege illegally. Usually these counterfeiting is mostly used by hackers to attacks.

6) The lack of self-management

Finally, but most importantly, manually maintaining these issues will be a very complex task, especially when the system is huge. The efforts paid to manage the explosive growth of information will overhead the capabilities of administrator. Thus, the system needs to find these problems and has to solve these problems by itself rather than administrators. In this article, we will introduce the selfmanagement techniques proposed by IBM to achieve adaptive [5]. The parameters of system could be adjusted dynamically based on changes in the external environment with the purpose of self-management. Such as data input with a low security requirement can be stored without encryption, but high-security data must be encrypted before storing. 


\section{Practical Solution}

The problems existing in university human information system can be resolved through scientific managements, appropriate technologies and strengthening trainings. Finally it will achieve our aims and ensures system to operate efficiently. The solution structure is shown as in Figure 1.

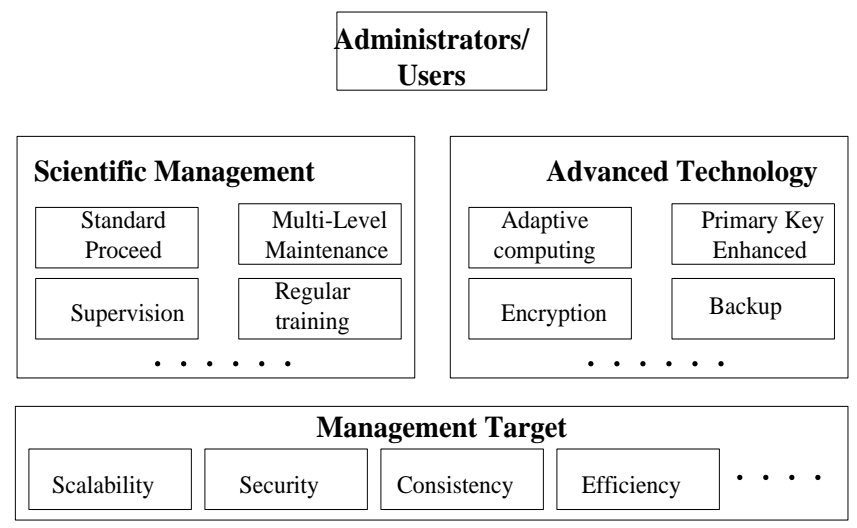

Figure 1. The Solution of Adaptive UHRIS

\subsection{Scientific Management Measures}

\section{1)Regulated data management processes}

When data is input, transformed, processed and stored and so on, there must be standard processes and steps of operations. Then operation guide is provided in details for users and each is responsible for correctness of data with necessary data constraints to reduce the errors probability. So we can strengthen data error control from every data source.

\section{2) Multi-Level maintenance}

In the university human resource system, it covers all the teachers, external staff, or short-term service person and etc. and provides data for many business departments such as organization department, the united front work department, trade unions and so on. The different departments need different data. So the operation and maintenance permissions should be assigned in multi-levels with requirements. Moreover, the high-level users should supervise the data provided by low-level ones. It can find non-standard data, incorrect data, and abnormal data and so on timely. These data should be submitted after modification.

\section{3) Regular training}

Through periodic technical training, it can improve user's professional skills, the work efficiency and quality of all levels in the system. And at the same time, all shortcomings found in the last periods during operating can also be changed to improve data quality.

\subsection{Technical Measures}

\section{1) Adaptive computing}

The aim of Adaptive computing is to design computing systems capable of awareness and self-management with minimum human intervention to handle complexity and uncertainties [6]. Adaptive computing analyzes system's state, actions and execution environment, determines the current situation, and triggers right execution components or mechanisms to keep and improve the self-management ability. Adaptive computing has 
been widely used in domain of data center, aerospace control and cloud computing system. In this paper, we will use it to improve the self-management ability of UHRIS.

2) Enhancing master data

Master Data, such as personal basic information, play an important role and is relied on by key application functions and decision support functions. Master data can be implemented by artificial means or specialized professional supporting tools to strengthen management, standardized testing. Such will ensure the accuracy of all master data. The quality of these master data decides the reliability of the key indicators during operating on some extent.

\section{3) Refining data}

When the data is defined and explained, we should make clear data meanings, value ranges and data relations and so on. The same data must be guaranteed from the same sources and the master data should be shared among different business functions. The irregularity data such as data types substandard, data value overflow, invalid data, conflicting with business rules and etc. must be cleared out through system testing or data cleaning tool. It should ensure proper correspondence between data and applications. Users should be refused when the usage of data for applications not supported or not compatible [7].

\section{4) Adoption of sound security technologies}

To ensure data security by strengthening the password strength, restricting error login, limiting authority, data encryption, server security testing, among other measures. Meanwhile, by full use of current advanced technologies of data storage, data can be backed up and recovered to avoid data dropping and failing, ensuring the normal operations of the system while some failures happen.

\section{The Architecture of UHRIS}

Following the principles proposed in last section, the human resources data management system in colleges and universities was designed by adaptive computing. It can be divided into general management module and self-management module. The former module includes staff management, personnel management, compensation management, training management, system management, recruiting management and so on, which just likes the common content of current human resources management systems. And the self-management module is used to maintaining general management module in a manner without human interruptions.

\section{1) Self-management module}

Self-management module is the basis for the adaptation of data management system, whose structure is shown as in figure 2. Self-management module consists of three-parts, sensor, analysis and execution, and MDR denotes for general management module. Sensor collects administrator's polices, users' features and the states of MDR, and the information is pushed into an information queue after being regulated. The information from the queue is put up to analysis module. Analysis module filters them first, and finds the right policy in the knowledge base, then calls the function analysis in turn, generating a series of service orders queue to control execution module. Execution module mainly implements specific methods to complete the management of MDR. Sensor, analysis, execution and knowledge base to form a feedback control loop for adaptive computing. 


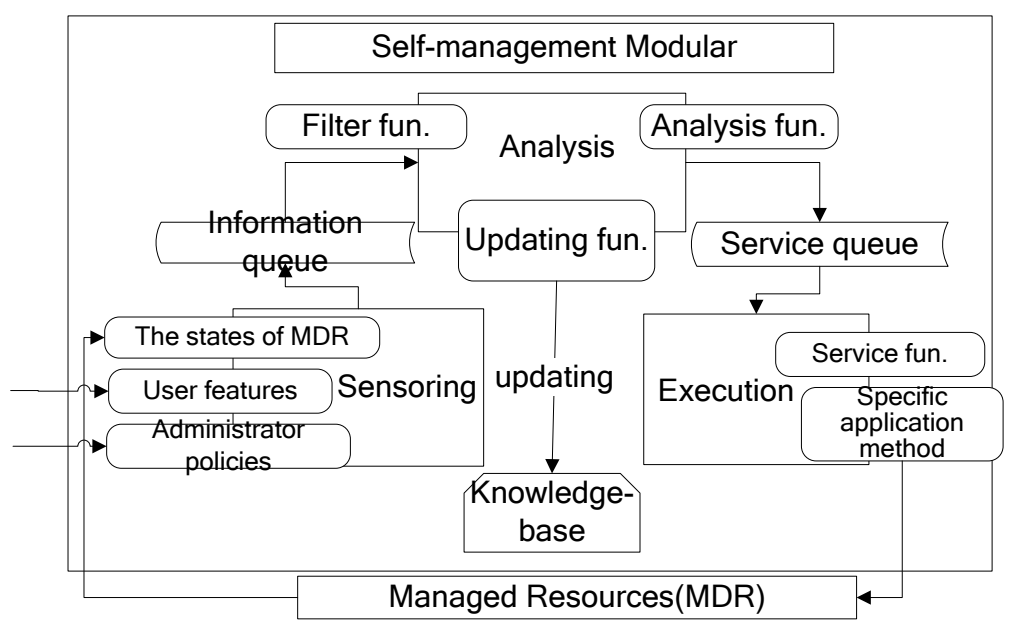

Figure 2. The Architecture of Self-Management Module

2) General management module

The architecture of general management module is designed as in Figure 3. Cryptographic module, recovery and consistency test modules are added to the new UHRMS, in which cryptographic modules mainly uses the algorithms of ADS or MD5 to encrypt the personal information stored. Recovery module is responsible for backing up important information and recovering them when needed; as well as consistency test module ensures the consistency of operation.

In the architecture, the improvement measures mentioned in the third section is specially designed, such as training data table as shown in Table 1. From the table, tenured training of teachers was demonstrated, and entire career training process can be recorded [8].

Table 1. The Table of Training Information

\begin{tabular}{l|l|l|l}
\hline Items & Type & Length & Description \\
\hline training_id & int & 4 & the identical number of training \\
\hline training_title & varchar & 50 & The title of training \\
\hline training_by & varchar & 50 & Training institution \\
\hline training_to & varchar & 50 & The group of people who should attend the training \\
\hline Content & varchar & 2000 & Introduction of the training \\
\hline [time] & varchar & 20 & The beginning and end time of the training \\
\hline
\end{tabular}




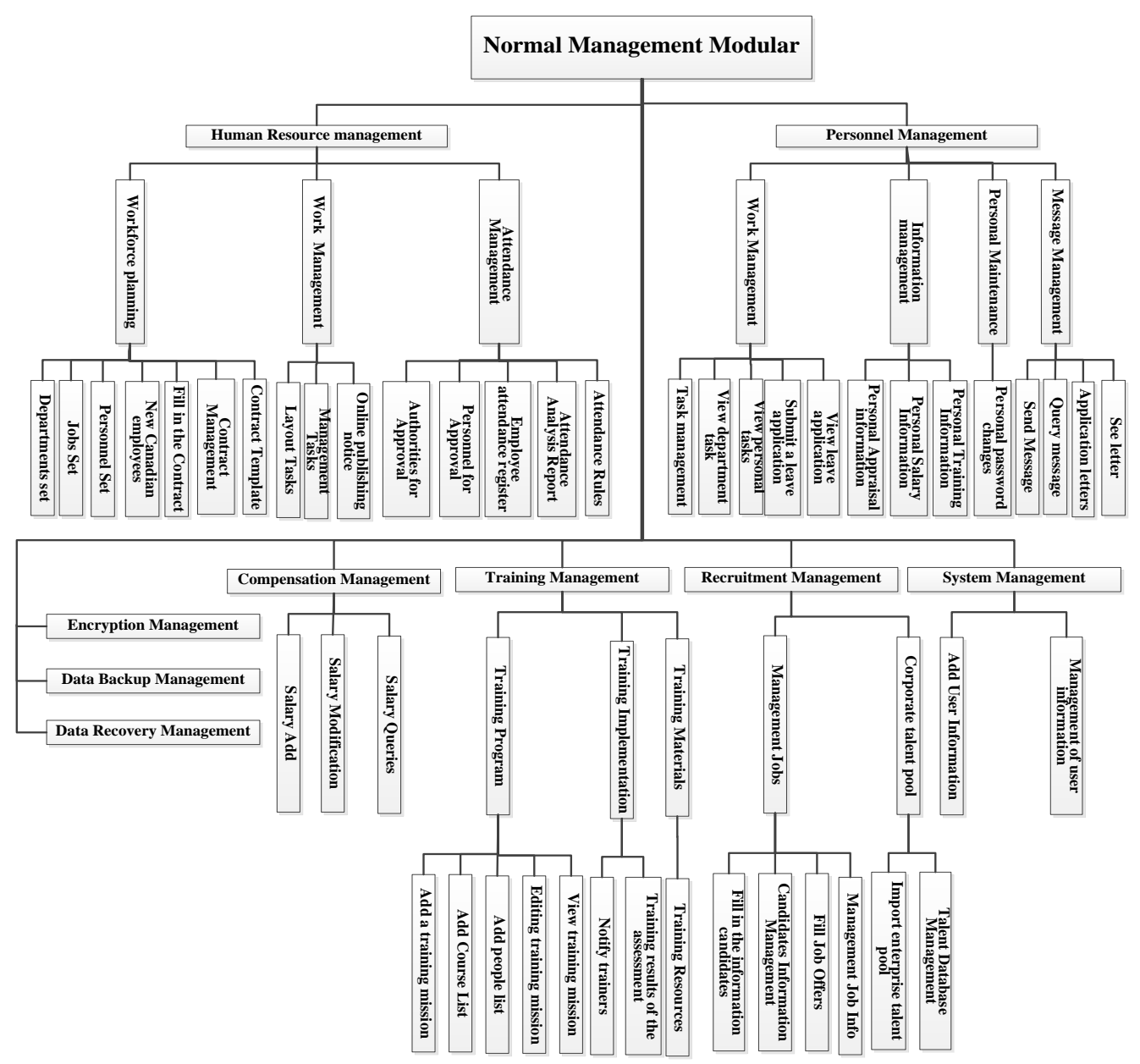

Figure 3. The Architecture of General Management Module

\section{Simulation and Analysis}

For analyzing the adaptive capacity of human resources data management system proposed in this article, we compared it with a conventional system using a performance evaluation tool, Load-Runner [9].

1) Experimental environment

To analyze the performance of adaptive UHRMS system, we have done some experiments about quality of service in a distributed computing application. These experiments are designed on the use of Load-Runner which tests service press of each node in environment. By comparing application within adaptive capability and without it, we can gain the differences in average response time and server throughput. Experimental environment uses a LAN network with a B/S mode. The traditional UHRMS system used for comparison is realized by closing the self-management module with the same system we proposed. Meanwhile, the processes that customers access the UHRMS system are simulated by Load-Runner.

2) Experimental results and analysis

The testing consist of 3 affairs, logging affair L_A, action affairs T_A, T_B. Affair L_A includes two stages, login and logout, which only run only once by each virtual user in its life cycle. T_A, T_B are a matter of iterative affairs happens repeatedly. To simulate different user, T_A, T_B are executed by $30 \%$ and $70 \%$ individually. There are 70 virtual users in the experiment, and the system simultaneously adds pressure to test the server 
quality by adding or closing the connection from virtual users. The stress experiment is designed refers to three loading stages, the pressure increasing stages, the pressure maintenance stage and the pressure releasing stage, just as in Figure 4.

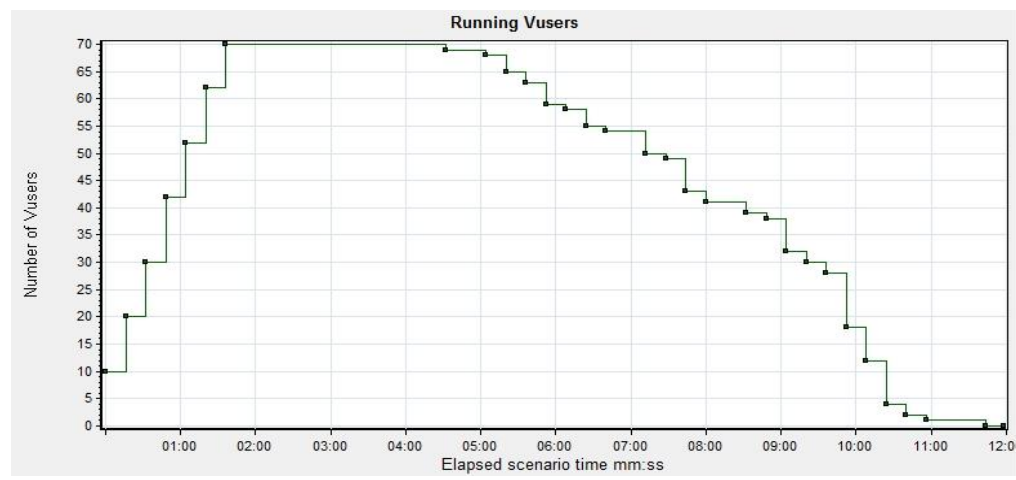

Figure 4. The Loading of Experiment

Adaptive UHRMS system is more suitable for concurrency transaction than ordinary ones in a B/S mode, because the load can be dynamically deployed using adaptive computing and it can timely adjust to environmental changes. For example, when a lot of teachers connect the server at the same time, the system reconfigures database connection pool to be maximum number of connections and optimize the service request queue to accommodate multi-user concurrent access. By stress testing, the performance of both average transaction response time and server throughput is summarized as below in Figure 5 and Figure 6.

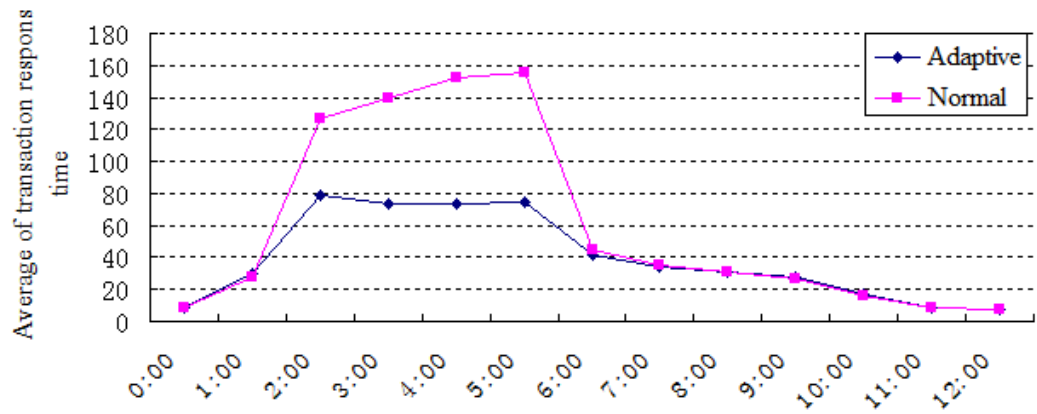

Figure 5. Comparison on the Average of Transaction Response Time

Average of transaction response time is an important evaluation metric for information system. It indicates whether an UHRMS system has great power to process an amount of concurrency transaction, which directly determines the customers' satisfaction of service in a huge distributed system. Seen from Figure 6, at first the average response time of adaptive UHRMS is more than normal UHRMSs', since the self-management module brings more works. But with workload increase continually, the response time of adaptive UHRMS is less than its competitor. And in whole, the average transaction response time of adaptive UHRMS is 39.1 seconds while the normal one is 60 seconds. The experiment results tell us adaptive environment significantly reduced response time of UHRMS and improved its quality of service. 


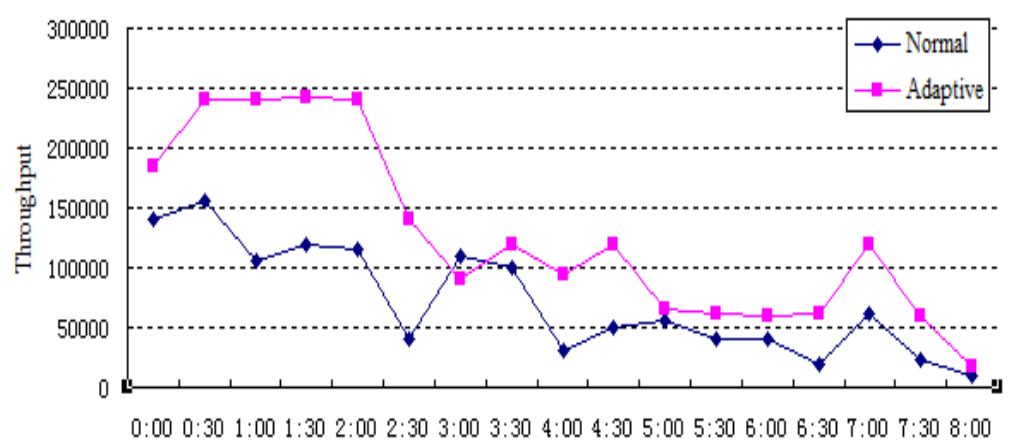

Figure 6. Comparison on the Average of Throughput

Throughout means the amount of work that a system deals with in a particular period. It determines the amount of service that clients can get per unit time. In Figure 6, the average throughput of adaptive UHRMS is always more than that of normal UHRMSs', and has a maximum throughput to be $2.1 * 106$ Byte.

From the above two figures, it is easy to find our adaptive UHRMS get a better performance than the traditional UHRMS. Besides, it is needed to point that the loading is designed in a stochastic manner in this test scenario, but it can also be any other type, and we can get a similar result of simulation.

\section{Conclusion}

In this paper, a new adaptive university human resource data management system is proposed to deal with current shortcoming exiting in traditional UHRMS, especially the complexity of management confronted by administrators. Moreover, the feature of scientific managements, appropriate technologies and strengthening trainings are also considered while designing and building the system. The experiment results show that adaptive UHRMS can greatly increase throughput as well as decreasing the average response time.

Meanwhile, data management is a long-term, continuous improvement process, it is wrong to think that all things can be done well only once. It requires long-term persistence and makes the necessary adjustments according to the changes in the specific circumstances [10].

\section{Acknowledgements}

This paper is a stage achievement of the project (f201217) of Natural Science Foundation of Heilongjiang Province and the project (12531175) of the Heilongjiang Province Education Bureau. Many people have contributed to it. This paper will never be accomplished without all the invaluable contributions selflessly from my group members.

\section{References}

[1] K. Ball, “The Use of Human Resource Information Systems: A Survey”, Personnel Review, vol. 30, no. 6, (2001), pp. 677-693.

[2] A. R. Hendrickson, "Human Resource Information Systems: Backbone Technology of Contemporary Human Resources", Journal of Labor Research, vol. 24, no. 3, (2003), pp. 381-394.

[3] C. Avgerou, "Information systems in developing countries: a critical research review", Journal of Information Technology, vol. 23, no. 3, (2008), pp. 133-146.

[4] D. Blumenthal, "Stimulating the adoption of health information technology", New Engl J Med., vol. 360 , (2009), pp. 1477-9.

[5] R. Sterritt, M. Parashar, H. Tianfield and K. Unland, "A concise introduction to autonomic computing", Advanced Engineering Informatics, vol. 19, (2005), pp. 181-187. 
[6] Y. Brun, G. Marzo Serugendo and C. Gacek, "Engineering Self-Adaptive Systems through Feedback Loops. Berlin", Heidelberg: Springer-Verlag, vol. 5525, (2009), pp. 48-70.

[7] Y. Bao and X. Qi, "Research on Data Quality of Large-scale Software System", Computer Engineering and Design, vol. 3, no. 32, (2011).

[8] D. Lei and S. Hui-zhang, "Research on Pipelining Management Mode of Human Resource System Based on B/S", Journal of Tianjin Normal University (Natural Science Edition), vol. 4, no. 30, (2010).

[9] HP LoadRunner software, Available at: https://h10078.www1.hp.com/cda/hpms/display/main/hpms_content. $\quad$ jsp?zn=bto\&cp=1-11-12617\%5E8_4000_100.

[10] D. Feng, M. Zhang and H. Li, "Big Data Security and Privacy Protection", Chinese Journal of Computers, vol. 1, no. 37, (2014).

\section{Authors}

Ye Fan, born in 1972, Master, research associate. Her research interests include human resource system and file management.

Shaoyun Guan, born in 1973, Master, associate professor. Her research interests include information system integrate and decision support and data security.

Honglue LV, born in 1970, Master, lecturer. His research interests include data mining and image recognition. 
International Journal of Database Theory and Application Vol. 8, No. 1 (2015) 